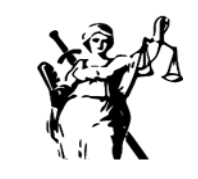

JUSTICIA

ISSN impreso 0124-7441
ISSN digital 2590-4566

\title{
Inclusión social y discapacidad asociada a los accidentes de tránsito.
}

\section{Social inclusion and disability associated with traffic accidents}

\author{
(iD) \\ Sandra Patricia Duque Quintero \\ Universidad de Antioquia, Colombia \\ spatricia.duque@udea.edu.co.
}

\author{
(iD) \\ Flor Patricia González Sánchez \\ Universidad de Antioquia, Colombia \\ fpatricia.gonzalez@udea.edu.co
}

\author{
(iD) \\ Sandra María Martínez Monsalve \\ Universidad de Antioquia, Colombia \\ sandra.martínez@udea.edu.co
}

Recibido: 07 de noviembre de 2019 / Aceptado: 9 de enero de 2020 https://doi.org/10.17081/just.25.37.3597

\begin{abstract}
Resumen
En este artículo de investigación se propone abordar el concepto de inclusión social como un elemento clave para el análisis de acciones encaminadas a propiciar espacios de inclusión para la población con discapacidad asociada a accidentalidad vial. La eficacia jurídica permite hacer énfasis en lo subjetivo, lo vivencial y lo cultural para identificar y comprender los desafíos jurídicos, esta fue planteada desde el enfoque de investigación de tipo cualitativo, para abordar la discapacidad asociada a la accidentalidad vial y determinar qué se aplica y cómo se logra el objeto de aplicación, entendiendo la problemática como un proceso histórico a partir de lógicas y con una mirada crítica al interior de las particularidades. De esta forma, y tras la pesquisa realizada, se pudo evidenciar que hablar de inclusión social implica el abordaje del concepto de exclusión social, por tanto, este artículo parte de los antecedentes de la exclusión social, lo cual conlleva la exploración de asuntos como: pobreza, desigualdad, protección social y riesgo social.
\end{abstract}

Palabras clave: accidentes de tránsito, discapacidad, exclusión social, inclusión social.

\section{Abstract}

In this research article it is proposed to address the concept of social inclusion as a key element for the analysis of actions aimed at promoting inclusion spaces for the population with disabilities associated with road accidents. Legal effectiveness allows the emphasis on subjective, experiential and cultural aspects to identify and understand legal challenges. This was proposed from a qualitative research approach, to address the disability associated with road accidents and determine what applies and how the application object is achieved, understanding the problem as a historical process based on logic and with a critical look inside the particularities. In this way, and after the investigation carried out, it was possible to show that talking about social inclusion implies the approach to the concept of social exclusion, therefore, this article starts from the background of social exclusion, which entails the exploration of issues such as: poverty, inequality, social protection and social risk.

KEYWORDS: disability, social exclusion, social inclusion, traffic accidents.

\section{Como citar:}

Duque Quintero, S. P., González Sánchez, F. P., \& Martínez Monsalve, M. M. (2020). Inclusión social y discapacidad asociada a los accidentes de tránsito. Justicia, 25(37), 167-184. https://doi.org/10.17081/just.25.37.3597 


\section{INTRODUCCIÓN}

Colombia definió la seguridad vial como uno de sus pilares en materia de política pública y esto se materializó con la expedición del Plan Nacional de Seguridad Vial 2011-2016, regulado en la Resolución 1282 de 2012 que buscaba, primordialmente, la reducción en un 50\% en la mortalidad generada por accidentes de tránsito. Esto se fundamenta en que la Organización Mundial de la Salud ha sustentado que los aumentos en los accidentes de tránsito ocupan el tercer nivel de las muertes en el mundo y que el aumento en las lesiones está generando discapacidad asociada al accidente. Ante esta realidad, se pregunta entonces, qué es un accidente de tránsito, definición que según el artículo 2 de Código Nacional de Tránsito es un evento generalmente involuntario, generado al menos por un vehículo en movimiento, que causa daños a personas y bienes involucrados en él e igualmente afecta la circulación normal de los vehículos que se movilizan por la vía o vías comprendidas en el lugar o dentro de la zona de influencia del hecho.

Posteriormente, se cambia el Plan inicial 2011-2016 por el Plan Nacional de Seguridad Vial 2011-2021, que fue regulado en la Resolución 2273 de 2014, amplía el periodo de observación de la iniciativa y detalla los pilares estratégicos, los programas y las acciones a seguir en materia de gestión institucional, comportamiento humano, atención-rehabilitación de víctimas, infraestructura y vehículos.

Según el anterior Plan Nacional de Seguridad Vial (2011-2016) - PNSV- todos los grupos de usuarios de la vía son vulnerables y puntualizaba que las cifras indicaban que los motociclistas y los peatones son quienes

aportan el 70\% de las muertes en siniestros de tránsito. Además, entre los años 2005 y 2010, se registró un incremento de fallecidos en accidentes de tránsito, el cual pasó de 5.418 a 5.502 y en el año 2010 se registraron más de 39.275 lesionados de gravedad según Medicina Legal, convirtiéndose los accidentes de tránsito en la primera causa de muerte en niños entre los 5 y los 14 años, y la segunda entre las personas de 15 y 24 años de edad (p.p.4-5).

En la metodología del PNSV (2011-2016) se planteó la matriz de HADDON la cual fue creada por el epidemiólogo William Haddon en 1970, este instrumento contiene dos dimensiones en materia de accidentalidad vial: 1. las fases (pre-durante y post) y 2. los factores intervinientes (sujetos-vehículo-vía). En el PNSV (2011-2016) se plantean las principales causas de siniestros de tránsito

la imprudencia del conductor, la desobediencia a la señalización, la pérdida de control del vehículo, o la presencia de alcohol en el conductor por mencionar sólo algunas. Estas causas están asociadas al factor humano, principalmente al comportamiento del conductor, sin embargo no son las únicas, y se distinguen también otras causas como las fallas mecánicas o deficiencias viales que están insertas en el factor vehicular y en el factor vial respectivamente...También podemos distinguir en cuanto al momento en que se producen; antes de que se produzca el siniestro (prevención del accidente), en el momento en que se produce (disminución de las lesiones y daños materiales) o finalmente después de que el siniestro de tránsito ha tenido lugar (conservación de la vida) (p. 5).

Los aspectos que han sido ligados a los accidentes viales deben ser relacionados para conjurarlos debido a que comportan el mismo núcleo esencial de protección y prevención. A propósito, Heinrich (1931) sustenta que se parte del sujeto y del ambiente en que fue educado, porque son eminentes los riesgos debido a las fallas personales y de mantenimiento que pueden originar el accidente vial y desencadenar la muerte o la lesión en la vía. Se ha reconocido que el sujeto en la vía está sometido a complejos requerimientos del sistema que pueden desbordar su capacidad para procesar la información que recibe, esto 
llama la atención en el sentido de que se debe propiciar que el sistema vial genere tranquilidad y seguridad para que se minimicen los riesgos y se controlen los accidentes viales. Concretamente, en el PNSV (20112016) se reguló la relación entre las diferentes fases y factores que muestran la orientación del modelo de Haddon en materia vial y muestra que se deben formular estrategias para suprimir los factores que condicionan y desencadenan accidentes, por lo que se requiere controlar la generación de riesgos presentes y futuros para establecer mecanismos de protección para los sujetos expuestos a ellos. No obstante, si bien los planes nacionales de seguridad vial se estructuraron por medio de pilares estratégicos en los que se plantean programas y acciones como la gestión institucional; el comportamiento humano; la atención y rehabilitación de víctimas; la infraestructura y vehículos, es preocupante la inclusión social que requieren las personas con discapacidad en Colombia, y en este caso específico, de aquellos cuya discapacidad está asociada a los accidentes viales. Es por esto que en este artículo se analizan los conceptos de inclusión y exclusión social para determinar la posibilidad real y material de la participación social de esta población. Igualmente, se realiza un análisis sobre la problemática de la discapacidad asociada a la accidentalidad vial.

\section{METODOLOGIA}

La eficacia normativa fue planteada desde el enfoque de investigación de tipo cualitativo, lo que permitió el abordaje sobre la discapacidad asociada a la accidentalidad vial, la recolección y el análisis de datos fundamentados en conceptos, el análisis de los datos, la denominación de sus categorías, la formulación de preguntas, la elaboración de comparaciones, la extracción de esquemas y la integración de los datos. Lo que se buscó con este enfoque fue el análisis sobre la normatividad y los instrumentos y determinar qué se aplica, es decir, determinar qué tanto logra su objeto de aplicación. Según Bobbio (2007) la eficacia de la norma se refiere a la "aplicación de las normas jurídicas...al terreno de los comportamientos efectivos de los hombres que viven en sociedad, de sus intereses opuestos, de las acciones y reacciones frente a la autoridad..." (p. 25)

La investigación se enfocó, en un acercamiento a la comprensión de la realidad jurídica de la accidentalidad vial por medio de la normatividad sobre la discapacidad en Colombia, entendiendo dicha realidad como un proceso histórico a partir de lógicas y con una mirada crítica al interior de las particularidades. La eficacia jurídica nos permite hacer énfasis en lo subjetivo, lo vivencial y lo cultural para identificar y comprender los desafíos jurídicos que se presentan en materia de planeación, implementación y evaluación de la normatividad sobre la discapacidad y su relación con los planes de seguridad vial. El objetivo de la investigación fue analizar la eficacia de la normatividad vial y la inclusión de la población con discapacidad asociada a los accidentes de tránsito en la ciudad de Medellín, lo que posibilitó realizar dos acercamientos: un rastreo documental desde los conceptos de accidentalidad vial, discapacidad, lesiones asociadas a los accidentes de tránsito; y el análisis de los Planes Nacionales de Seguridad Vial 2011-2016 y 2011-2021 y el Plan de Movilidad Segura de Medellín (2014-2020); artículos de revista, programas de capacitación vial, jurisprudencia y legislación. 


\section{RESULTADOS DE INVESTIGACIÓN}

\section{Descripción de los conceptos exclusión social e inclusión social}

Los sujetos, dadas sus condiciones han sido clasificados de acuerdo con algunas especificidades que forman parte de las transformaciones del mundo de lo social, por ejemplo las categorías de habitante en situación de calle, adulto mayor, proletario, discapacitados, son parámetros establecidos para su desenvolvimiento en la sociedad, pero estas categorías sociales abarcaban diversos grupos y problemáticas, lo que permitió la generación de medidas de protección social para lograr la inserción de quienes, por sus condiciones socioeconómicas, se encontraban en situación de desventaja, vulnerabilidad o marginalidad. Por tanto, la protección social generalmente se ha definido como aquellas "medidas del sector público para proveer seguridad de ingresos a las personas" (Holzmann \& Jorgensen, 2003, p.2); pero esto representa una mirada tradicional del concepto el cual se transforma de forma concomitante con los retos económicos y sociales que presentan los países y que deben ser atendidos mediante la cooperación de instituciones económicas como el Banco Mundial o la OCDE.

En vista de las limitaciones de esta visión tradicional se privilegia el papel del sector público, pero se pasa por alto sus efectos positivos en el desarrollo económico por cuanto hace énfasis en asuntos de gastos y costos, y porque se clasifican las intervenciones en programas sectoriales sin un enfoque estratégico con impacto en la reducción efectiva de la pobreza para integrar a quienes necesitan debido a que no participan en el proceso de crecimiento, por lo que se considera que "...se desarrolla una nueva definición y marco conceptual llamado manejo social del riesgo, que debería permitir un mejor diseño de programas de protección social como un componente de una estrategia revisada de reducción de la pobreza" (Holzmann \& Jorgensen 2003, p. 4). Bajo esta perspectiva, se observa que la situación que afecta a un grupo poblacional, permite su visualización cuando la protección social se asume como un tipo de inversión. La idea es contribuir a que haya acceso a los servicios sociales básicos y se evite la exclusión social.

El manejo social del riesgo se basa en la idea fundamental de que todas las personas, son vulnerables a múltiples eventos de diferentes orígenes, ya sean naturales, como terremotos, inundaciones y enfermedades o producidos por el hombre, como el desempleo, el deterioro ambiental y la guerra. Lo que muestra vulnerabilidad entendida como "un proceso de malestar producido por la combinación de múltiples dimensiones de desventaja, en que toda esperanza de movilidad social ascendente, de superación de su condición social de exclusión o próxima a ella, es contemplada como extremadamente difícil de alcanzar" (Hernández, 2011, p. 201). Los eventos y riesgos que afectan a las personas y comunidades en algunas ocasiones no se pueden evitar, por lo tanto, generan y profundizan la desigualdad social porque induce a que las personas estén más expuestas a riesgos al no contar con los instrumentos adecuados para su manejo. (Holzmann \& Jorgensen, 2003, p. 4-5)

En concordancia con lo anterior, la articulación entre el manejo social del riesgo y la protección social conllevan la necesidad de identificar, seleccionar y suministrar elementos o herramientas para reducir la vulnerabilidad y para brindar instrumentos para el reconocimiento de las necesidades, potenciando las capacidades y brindando oportunidades. Bajo estas premisas, es importante consolidar políticas económicas, ejercer con responsabilidad el poder y ampliar el acceso a la salud y a la educación básica.

Se considera que el concepto de exclusión social se ha relacionado con problemas de desempleo e inestabilidad de los vínculos sociales en un contexto que involucra nuevos actores y otras dinámicas sociales que pueden verse a partir del estudio de la pobreza como enfoque lo que permite analizar asuntos como la exclusión, la vulnerabilidad, la acumulación de desventajas, la segregación residencial y el empobrec- 
imiento, lo que posibilitaría la mirada a otros fenómenos relacionados con la pobreza. En el análisis de la "Nueva pobreza" se identifican dimensiones como los mercados de trabajo, la segmentación de los servicios sociales básicos, la dimensión del consumo, la movilidad social, la territorialidad de la pobreza y las materias relacionadas con las pautas asociativas de las familias en situación de pobreza. Todo lo anterior abre el panorama para la comprensión del fenómeno por lo que se analizan aspectos como la marginalidad que según Espinoza \& Francisco (2008) está constituido por "un contingente de personas excluidas de las sociedades y otras tantas vulnerables a la exclusión" (p.4); la internacionalización de la pobreza en articulación con el proceso de globalización; la composición social, lo cual implica visibilizar el fenómeno de la pobreza teniendo en cuenta la heterogeneidad de grupos sociales y otras categorías.

Una referencia fundamental para abordar el concepto de exclusión social, es representada también por el filósofo y economista indio Amartya Sen (2000) en su texto "El desarrollo como libertad", que a partir de sus estudios sobre las condiciones de la pobreza abordadas sustenta cómo el grado de privación de una persona en condición de pobreza, incide en el desarrollo pleno de sus capacidades, y esto a su vez, interfiere en su libertad pues esa condición impide el acceso a la nutrición, a la salud, al abrigo y al techo, aspectos necesarios para la supervivencia. Adicional a esto, considera Sen (2000, p. 15) que el orden en el escenario de lo local, la participación en la vida social, política y económica se ven amenazados por la condición de vulnerabilidad en que se encuentran quienes están en la pobreza. En esta lógica, los estudios empíricos han permitido identificar diferentes tipos de libertad con una visión instrumental, Sen (2000) considera que son las "libertades políticas, facilidades económicas, oportunidades sociales, garantías de transparencia y redes de seguridad" (p. 19), cada uno de estos elementos deben contribuir en el mejoramiento de las capacidades humanas. Por tanto, para Sen (2000, p. 19) la articulación entre políticas públicas para impulsar las capacidades humanas y las libertades con la promoción de las libertades instrumentales, pueden ser una estrategia de discusión y apropiación de estas libertades para que sean motor de desarrollo.

Los planteamientos anteriores permiten complejizar el concepto de exclusión social desde un conjunto de acciones sociales encaminadas al bienestar de las personas, que representa un campo analítico donde convergen diversos saberes, no solo de las ciencias sociales, sino también de las ciencias exactas, este relacionamiento permitiría comprender contextos de índole político, social, ideológico e institucional en el cual se gesta el bienestar para la construcción de nuevos escenarios de inclusión social donde las personas y los grupos sociales cuenten con oportunidades para vivir con dignidad. Así las cosas, es visible la interrelación entre políticas sociales, políticas públicas, dinámicas del mercado, bienestar individual y colectivo, objetivos, metas y resultados.

En la actualidad, los debates sobre exclusión social son vigentes en los discursos académicos y en la sociedad lo cual permite que se discuta, debata, interrogue y construya con base en nuevas realidades. Para Camacho $(2014$, p. 209) comprender el fenómeno de la exclusión social reviste una aprehensión compleja ya que la integran dimensiones que se encuentran interrelacionadas entre sí, se traduce en la acumulación y persistencia de problemas y carencias en multitud de ámbitos, supone la falta de acceso a recursos y necesidades básicas por lo que no se cumple con una plena inserción social y esto implica una falta de participación en la dinámica social. Lo que permite que se minimice una mirada centrada exclusivamente en lo económico enmarcada como carencias o insuficiencias de ingresos, para centrar la atención en la integración social.

El concepto de inclusión social se puede considerar como una estrategia para atenuar las problemáticas que trae consigo la exclusión social, y en ese sentido cobra importancia el hecho de que, para comprender este fenómeno, precisamente se estudie lo que ha significado su contrario, es decir, la exclusión. Entonces, un concepto comporta el otro; es más, tanto la exclusión como la inclusión social son fenómenos dinámicos 
generados por una variedad de factores multidimensionales que complejizan su análisis, como el mercado, la democracia y el estado de bienestar. En esa misma medida, el término de inclusión social es amplio pues en sí se pueden encontrar políticas de empleo, pobreza, distribución de la tierra, acceso a servicios, discriminación, género, ciudadanía e interculturalidad, discapacidad, entre otras, y todos están comprendidos en el concepto de inclusión social. De esto se puede inferir que el término sirve para aunar situaciones de diferente indole, y, por tanto, también los discursos de los actores que intervienen desde sus subjetividades son diversos. En todo caso es importante resaltar que si bien la inclusión social surge en el contexto de la exclusión, ambos conceptos posibilitan la identificación de categorías de análisis, incluso en el concepto de inclusión social se ha buscado atacar problemas que no están en el ámbito de la exclusión y que aun así no dejan de ser problemas, por ejemplo la "falta de participación y poder político, desempleo juvenil, desempleo y precariedad del empleo, racismo, intolerancia cultural, explotación económica..." (Young, 2000, Citado por Sandoval, 2016, p. 72).

La inclusión social hace referencia a que todas aquellas personas que se consideran que están por fuera de la sociedad deben ser atraídas para que formen parten, y participen. Esta visión hace pensar en términos binomios de incluido - excluido, dentro - fuera, centro - periferia o margen, lo que dificulta la comprensión del término. Aunado a esto, la inclusión social ha estado asociada a términos como integración social, cohesión social o capital social, entre otros, aristas que permiten múltiples análisis. Por lo que se parte de entender que hay personas por fuera de la sociedad, aunque esto es impreciso, porque las personas, aunque estén al margen siguen formando parte de una sociedad debido a que genera interacciones y transacciones con las instituciones y las organizaciones desde otras lógicas,

Como se evidencia, el argumento que sostiene la amplitud del concepto inclusión social se confirma cuando se sostiene que es un derecho social, o es un elemento de la estructura social, política o económica, o es una base ética de la cohesión social o hace parte de una idea de participación en la vida económica. Sin embargo, dadas todas esas aristas existe consenso en considerar que el objetivo general de este concepto es que una sociedad logre ser cada vez más inclusiva por medio de dos mecanismos claves: la redistribución y el reconocimiento. Para Sandoval $(2016$, p.87) estos mecanismos están sustentados en políticas, programas, planes, leyes, reglas, instituciones y otros instrumentos que administra el Estado de forma articulada para canalizar y cambiar la forma en que se distribuyen los recursos materiales (redistribución) y simbólicos (reconocimiento) a aquellos grupos que por falta de estos o por la negación sistemática participan en la sociedad de manera desventajosa o no significativa.

Para Sandoval (2016, pp. 85-86) esto es visible en problemas como la subestimación por hacer parte de ciertas culturas, los estereotipos y los prejuicios. Lo particular de estos tipos de injusticias es que se encuentran arraigados en todos los ámbitos de la sociedad, propiciando desventajas entre las personas dentro de las dinámicas sociales, culturales, cotidianas o públicas. Señalarlas sirve para indicar que a cada una atañe una solución. Por lo que se plantea que la redistribución permite generar cambios que conduzcan a la equidad y reconocimiento para lograr la reparación; es decir, comprender la diferencia y diseñar políticas encaminadas a valorar esa diferencia. Por tanto, ambas condiciones necesitan instrumentos como las reglas, las leyes y las políticas que permitan su implementación.

Por tanto, una forma de entender el proceso de inclusión social conlleva la incorporación de las personas o grupos en distintos mecanismos articulados o interdependientes de redistribución y reconocimiento, pues la participación en la sociedad de forma desventajosa o no significativa se debe principalmente a las reglas y normas (instituciones y cultura) y las carencias materiales y simbólicas, individuales o grupales, que estas reglas y normas producen. Además, esta idea es sustentada por Sandoval (2016, p. 91) sumando otras, como lo son las características individuales, como la falta de apoyo familiar y de redes sociales; 
también, la vejez, el género, la raza, las enfermedades crónicas, la discapacidad, la preferencia sexual, la pertenencia a una cultura diferente, entre otras, que al ser percibidas y valoradas desde las instituciones y la cultura dominantes resultan en una participación que es desventajosa para los sujetos y grupos en los distintos ámbitos de la sociedad. Con base en los acercamientos conceptuales descritos, y una vez expuestos los mecanismos de redistribución y reconocimiento, es importante mencionar que para comprender la inclusión social hay que determinar que las personas sí participan, construyen y se benefician de y en la sociedad, pero que esa participación también podría ser en condiciones desfavorables o desventajosas.

Cabe preguntarse entonces, quién o quiénes llevan a cabo los procesos de inclusión social. Es sabido que el Estado cumple un rol principal en la medida en que lidera e implementa políticas para dar respuesta a las demandas por medio de sus instituciones, desde el nivel ejecutivo, legislativo, judicial y entidades de control. Por lo que se administran los recursos, se redistribuyen y se ejecutan los mecanismos de reconocimiento, por su parte la sociedad civil, los sujetos, las familias, las empresas, el sector privado, los movimientos sociales y diversas organizaciones, proveen e inciden en el desarrollo de políticas de inclusión social, las cuales, para ser exitosas, deben estar compuestas de voluntad política, recursos económicos y capital cultural y simbólico. Como puede verse, las dinámicas de inclusión social necesariamente llevan a pensar en la vinculación de las políticas públicas a actividades enfocadas al desarrollo de la vida en sociedad. El papel del Estado se orienta en su capacidad para articular a los diversos actores sociales en el desarrollo de un proyecto concreto y aprovechar los liderazgos locales para desarrollar acciones desde una visión y unos objetivos estratégicos compartidos y definidos. Eso significa, para Sandoval (2016, p. 159) que los gobiernos deben asumir, más allá de la gestión de las propias competencias, un rol promotor de la articulación de los diferentes actores que intervienen en la acción social.

La creación de políticas públicas, desde una mirada tradicional, la entiende Lahera (2002) como la "acción de una autoridad pública o al resultado de la actividad de una autoridad investida de poder público y de legitimidad gubernamental" (p. 4). Sin embargo, este concepto muestra que los cambios y los retos en la sociedad tienen la posibilidad de que se utilicen múltiples formas de abordar los problemas colectivos y sociales dadas las nuevas tecnologías de la información y la comunicación que permiten mayor acceso a los datos y la participación ciudadana. Así las cosas, se puede decir que en las políticas públicas conllevan flujos de información y cursos de acción con objetivos claros definidos de acuerdo con los requerimientos de las comunidades. Una política pública sobre discapacidad asociada a accidentalidad vial, debe incluir, según Lahera (2002) “...orientaciones o contenidos, instrumentos o mecanismos, definiciones o modificaciones institucionales, y la previsión de sus resultados" (p.4). Es por esto que, al entender la exclusión social como un proceso de desintegración, donde se fragmentan o rompen las relaciones entre los individuos y la sociedad, las políticas públicas constituyen una oportunidad de acción e intervención en los procesos sociales, ya que cuentan con un proceso donde se evalúan las inversiones, los resultados y el impacto en los cambios sociales. De esta manera, las políticas sociales en Colombia marcan la pauta en las acciones que las instituciones encargadas de atender a la población deben cumplir. Una vez definidos los conceptos de exclusión e inclusión social, es importante adentrarse, para la comprensión del objeto de investigación, en el significado de la discapacidad, y así, caracterizar la discapacidad asociada a accidentes de tránsito.

\section{Análisis de la discapacidad asociada a accidentalidad vial}

A través de los diversos estudios sobre exclusión e integración social, se han logrado identificar ocho ámbitos en los cuales se puede clasificar este fenómeno: económico, laboral, formativo, sociosanitario, vivienda, relacional, político y de contexto espacial. Según la Fundación BBVA (2005) de cada uno de estos se estudia el espacio, el factor y los indicadores; sin embargo, para el caso concreto nos detendremos en describir 
algunos aspectos clave de cada uno y en identificar dónde se encuentra ubicado el tema de la discapacidad asociada a accidentalidad vial, para tener una idea acerca de las formas de exclusión a las cuales pueden verse sometidos las personas dentro del contexto social

Ámbito económico la discapacidad asociada a accidentes de tránsito ha sido tratada como un componente en el concepto de exclusión, es decir, se presenta evidencia de que se genera exclusión social desde las disparidades económicas en sectores de la sociedad; adicional a esto, es innegable que el ámbito económico incide en la mayoría de procesos relacionados con la exclusión que se puede predicar de esta discapacidad asociada a los accidentes viales en tanto convergen variables como: la calidad de arrendatario, niveles de ingresos insuficientes, dificultades financieras del hogar y desprotección social (Fundación BBVA, 2005, p. 39).

Ámbito laboral genera interés en la medida en que en la actualidad existe una lógica de competencia y de mercado que suscita una fragmentación e individualización lo cual incide en las relaciones laborales y ubica el trabajo en el centro de lo social ligado a una idea de "seguridad" que se ha ido transformando con el paso del tiempo concomitante a las demandas del mercado, pues lo que impera en estos momentos es la problematización sobre la estabilidad laboral dadas las nuevas formas de producción, tecnificación y relaciones contractuales que reconfiguran el mercado de trabajo y que repercuten en las regulaciones o no, independientemente que se enfoque en la flexibilidad y la adaptabilidad. Bajo esta óptica, en la discapacidad asociada a accidentes de tránsito se concentran dos formas de exclusión: una que tiene que ver con el acceso al mercado laboral donde se analizan aspectos como el desempleo, el subempleo, la descualificación y la imposibilidad; y otra que se deriva de las condiciones de trabajo donde se enfoca la mirada en la precarización del empleo (Fundación BBVA, 2005, pp. 54-55).

Ámbito educativo es un pilar fundamental de la sociedad, asimismo, a partir de este han surgido procesos de marginación. Es importante decir que la educación se encuentra dentro de un conjunto de factores de índole individual, socioculturales, políticos y económicos que participan en el desarrollo de sus procesos. Así, hay personas excluidas del sistema educativo, y otros, que por sus condiciones socioeconómicas y de recursos educativos se consideran dentro del fenómeno de la exclusión. En el caso del acceso al sistema educativo se resalta que se deben analizar factores como el analfabetismo, el fracaso escolar, el abandono del sistema educativo y las barreras lingüísticas y comunicacionales que están presentes antes y también después de una discapacidad asociada por accidentalidad vial.

Ámbito sociosanitario, la discapacidad asociada a accidentes viales se enfrenta al derecho a la salud, el cual se vulnera por los obstáculos que tienen las personas para acceder a una atención integral, y esto repercute negativamente en el desarrollo humano y en el mejoramiento de la calidad de vida de los sujetos. Los aspectos que se tienen en cuenta al momento de estudiar este ámbito son las lesiones, la mortalidad y la morbilidad. Y los sujetos discapacitados se clasifican en sistemas de estabilidad financiera del sistema de salud como generadores de dependencia y estigmatización social

Alteraciones estigmatizadas socialmente, los Trastornos mentales estigmatizados socialmente, las Enfermedades infecciosas estigmatizadas socialmente, las Enfermedades que provocan malformaciones, las Alteraciones de la imagen corporal, las Deficiencias que provocan una exclusión social institucionalizada por secuelas anatómicas y funcionales, y, por último, las Discapacidades graves que generan dependencia. (Fundación BBVA, 2005, p. 90)

Ámbito de la vivienda digna existe una estrecha relación con el espacio que se ocupa porque se puede manifestar la desigualdad y la exclusión social, esto se expresa en la ubicación de los colectivos en ciertas 
condiciones, por tanto, la vivienda, que hace referencia al espacio que se habita, es otra unidad susceptible de analizarse bajo la perspectiva de la accesibilidad y las condiciones de la vivienda que deben estar presentes en el caso de la discapacidad asociada a accidentes viales (Fundación BBVA, 2005, p. 104).

$\gg$ Ámbito relacional son importantes las relaciones familiares y sociales porque son la base para hacer frente a las contingencias por riesgos o vulnerabilidad y, en caso contrario, su ausencia o precariedad pueden llevar a la profundización del abandono o al descuido de los sectores excluidos como puede ocurrir en el caso de la discapacidad asociada a accidentalidad vial. De esta manera, las redes familiares y las redes sociales son elementos clave para conjurar la discapacidad. (Fundación BBVA, 2005, p. 118).

$\#$ Ámbito político es fundamental porque en este se da la inclusión y la participación social. Es aquí donde hay acceso o no a la defensa de los derechos económicos, sociales y políticos en materia de la discapacidad asociada a accidentes viales. (Fundación BBVA, 2005, p. 137).

$\gg$ Ámbito contexto espacial, se hace una segmentación en tres componentes que determinan el contexto espacial en el caso de la discapacidad asociada a accidentalidad vial: espacio físico, espacio sociocultural y espacio económico. En el primero, la exclusión social es visible por el deterioro en edificios, vivienda, servicios, espacios públicos y deficiencia en la movilidad. En el segundo, la estigmatización del territorio, la inseguridad ciudadana, la falta de cohesión social y la ausencia de recursos y equipamientos públicos. En el tercero, se revela la exclusión a partir de la marginación económica (Fundación BBVA, 2005, p. 151).

En los párrafos anteriores se abordaron los conceptos exclusión social e inclusión social ya que en materia de la discapacidad asociada a los accidentes viales se evidencian carencias, desventajas y limitaciones de ciertos sectores de la población, lo que muestra la afectación de la promoción social de los ciudadanos y el disfrute de los derechos fundamentales. Con base en la identificación de estas situaciones, surgen unidades de análisis para comprender y atender la vulnerabilidad que se genera con esta problemática particular. La Organización Mundial de la Salud (OMS) y el Banco Mundial en el informe mundial sobre la discapacidad (2011) consideran que la discapacidad forma parte de la condición humana "casi todas las personas sufrirán algún tipo de discapacidad transitoria o permanente en algún momento de su vida...La discapacidad es compleja, y las intervenciones para superar las desventajas asociadas a ella son múltiples, sistémicas y varían según el contexto" (p.7).

En este orden de ideas, existe una preocupación porque este informe revela que los discapacitados "casi 200 millones experimentan dificultades considerables en su funcionamiento" (OMS \& Banco Mundial, 2011, p.5). Pero esta preocupación no es reciente, porque en todas las épocas y sociedades hay prevención en materia de discapacidad e incluso, se han identificado tres modelos en el tratamiento social de este fenómeno: modelo de prescindencia, del que se desprenden dos submodelos, el eugenésico y el de marginación; el modelo rehabilitador o modelo médico y el modelo social (Toboso \& Arnau, 2008).

El modelo de prescindencia considera que el origen de una discapacidad es religioso y el sujeto discapacitado es una carga para la sociedad porque no puede ser productivo. El submodelo eugenésico se fundamenta en asuntos religiosos y políticos, se considera conveniente el desarrollo y crecimiento de los menores en condiciones de normalidad, para los griegos la discapacidad era resultado del pecado y para los romanos era la advertencia de que la alianza con los dioses estaba rota. Según Garlan (1995, citado por Toboso \& Arnau, 2008, p. 66) dadas estas condiciones y creencias se llevaron a cabo prácticas de eugenesia e infanticidio en esas sociedades. El submodelo de marginación tiene características históricas constantes; por ejemplo, en la edad media, las personas con discapacidad estaban incluidas dentro de los grupos de los pobres y los marginados (Toboso \& Arnau, 2008, p. 66); la exclusión se daba por temor o rechazo. Por lo 
que los sacerdotes y médicos decidían, dependiendo de la opinión teológica, si los discapacitados morían porque no podían suplir sus necesidades, o si sobrevivían en la mendicidad (Toboso \& Arnau, 2008, p. 66).

El modelo rehabilitador o médico tiene como característica que sus causas son determinadas a través de un ejercicio médico-científico, por lo que se considera como una "enfermedad" o "ausencia de salud". En este modelo la persona con discapacidad puede aportar a la comunidad en la medida en que sean rehabilitadas o normalizadas; por tanto, se lleva a cabo "un proceso de normalización" para obtener la connotación de ciudadanas o ciudadanos. Esto trae como consecuencia que el centro de atención sea la discapacidad, y no la persona, esta se subestima y el tratamiento es de corte paternalista enfocado en una supuesta enfermedad. Adicionalmente, como estas personas se convierten en "objetos médicos" son medicalizadas para ser normalizadas. Como, por ejemplo, la educación especial (Toboso \& Arnau, 2008, pp. 67-68).

El modelo social, surge para dar respuesta a los dos modelos anteriores aduciendo que las causas que originan la discapacidad son sociales "La utilización del término "social" pretende, en este caso, remarcar que las causas que originan la discapacidad no son individuales, de la persona afectada, sino sociales, por la manera en que se encuentra diseñada y construida la sociedad" (Toboso \& Arnau, 2008, p. 68). Quienes defienden este modelo consideran que la sociedad tiene limitaciones para prestar los servicios que las personas con discapacidad necesitan. Asimismo, se parte de la realidad de que todo ser humano es digno y puede aportarle a la sociedad desde la inclusión y la aceptación de la diferencia. En palabras de Toboso \& Arnau (2008, p. 68)

Estos presupuestos tienen importantes consecuencias, en las que se destacan sus repercusiones en las políticas sociales a ser adoptadas sobre las cuestiones que involucran a la discapacidad. Así, si se considera que las causas que la originan son sociales, las soluciones no deben dirigirse individualmente a las personas que se encuentran en dicha situación, sino hacia la sociedad. Si el modelo rehabilitador se centra en la normalización de las personas con discapacidad, el modelo social aboga por la normalización de la sociedad, de manera que ésta llegue a estar pensada y diseñada para atender las necesidades de todos.

En la sociedad y en el marco de las dimensiones normativas y éticas, el modelo social se aparta del tratamiento de la discapacidad solo bajo criterios médicos, y aunque no desconoce la realidad médica propugna porque las personas escojan su forma de vida. Por un lado, la ciencia debe aportar soluciones pertinentes para los asuntos relacionados con la salud, y, por otro lado, la sociedad debe promover la igualdad de oportunidades para evitar la exclusión. Este viraje en la forma de abordar el fenómeno de la discapacidad implica que, si alguna persona es discriminada por esta condición, este acto se considerará un atentado contra los derechos humanos. A propósito, a nivel internacional, Colombia firmó la Convención Nacional de derechos de las personas con Discapacidad el 30 de marzo de 2006, lo cual representa un compromiso de país, además es evidente que el concepto de discapacidad debe cambiar al igual que la percepción y la aceptación de la sociedad frente al fenómeno. Según la Convención su propósito es :

promover, proteger y asegurar el goce pleno y en condiciones de igualdad de todos los derechos humanos y libertades fundamentales por todas las personas con discapacidad, y promover el respeto de su dignidad inherente. Las personas con discapacidad incluyen a aquellas que tengan deficiencias físicas, mentales, intelectuales o sensoriales a largo plazo que, al interactuar con diversas barreras, puedan impedir su participación plena y efectiva en la sociedad, en igualdad de condiciones con las demás (Naciones Unidas [UN], 2006). 
Con base en lo presentado en los párrafos anteriores, se considera que la discapacidad ha ampliado su análisis debido a que se han generado nuevas categorías de análisis susceptibles de investigarse para comprender desde las subjetividades de las personas, esto permite asumir las lógicas de la discapacidad desde su abordaje y el tratamiento de quienes se encuentran en esta condición. Esto muestra que es necesario analizar lo que ocurre particularmente en el caso de la discapacidad asociada a la accidentalidad vial.

La Asamblea General de las Naciones Unidas presentó el programa titulado “Decenio de Acción para la Seguridad Vial 2011-2020", en el que Colombia participó y se adhirió en mayo de 2011. Sus objetivos están dirigidos a: contribuir en salvar vidas mediante el mejoramiento de la seguridad en la vía pública; determinar el comportamiento de los usuarios de la vía pública y evaluar los servicios de urgencias. Colombia definió la seguridad vial como uno de sus pilares en materia de política pública y se enfoca en la prevención de los accidentes viales porque deben ser considerados una pandemia que debe tratarse con estrategias efectivas de prevención. La accidentalidad y sus efectos son un creciente problema de salud pública que desproporcionada e inequitativamente afecta a los usuarios de las vías. (Cabrera, Velásquez \& Valladares, 2009, p. 219). Esto se fundamenta en que la OMS ha sustentado que los aumentos en los accidentes de tránsito ocupan el tercer nivel de las muertes en el mundo y el aumento en las lesiones está generando discapacidad asociada al accidente. Por accidente se entiende, según el Art 2.L769/2002, Código Nacional de Tránsito, un

Evento generalmente involuntario, generado al menos por un vehículo en movimiento, que causa daños a personas y bienes involucrados en él e igualmente afecta la circulación normal de los vehículos que se movilizan por la vía o vías comprendidas en el lugar o dentro de la zona de influencia del hecho.

La OMS consideró en el informe presentado a las Naciones Unidas en el año 2003 que “... cientos de miles más se lesionan en nuestros caminos, y algunos se convierten en discapacitados permanentes. La gran mayoría de esto ocurre en países en desarrollo, entre los peatones, los ciclistas, los motociclistas y los usuarios del transporte público..." (Organización Panamericana de la salud [OPS] \& Organización Mundial de la Salud [OMS], 2011). "Las proyecciones de la OMS indican que, para 2020, las lesiones causadas por accidentes de tránsito podrían ser la tercera causa de muerte y discapacidad, de la gravedad de otros problemas de salud como la malaria, la tuberculosis y el VIH» (Organización Panamericana de la salud [OPS] \& Organización Mundial de la Salud [OMS], 2004). Por estas razones se calculó que a 2020 la segunda causa de mortalidad y morbilidad en los países emergentes será debido a los accidentes asociados a la accidentalidad vial (Organización Panamericana de la salud [OPS] \& Organización Mundial de la Salud [OMS], 2004).

Con base en las proyecciones la mirada se enfoca en la construcción de estrategias multidimensionales para el tratamiento y rehabilitación de las personas que luego de sufrir lesiones de tránsito se ven afectadas por discapacidades asociadas a estos. Por lo que los factores psicológicos, las limitaciones funcionales, la participación, el contexto y la cultura, son las bases para el diseño de campañas y políticas de prevención para proyectar y planear herramientas ciudadanas que permiten disminuir los episodios de lesiones y de discapacitados asociados por la accidentalidad vial. La Clasificación Internacional del Funcionamiento de la Discapacidad y la Salud - CIF -, generó categorías de análisis y dominios de salud para describir ámbitos como el corporal, el individual, las funciones y las estructuras corporales. Se entiende por dominios el "conjunto relevante, y práctico de funciones fisiológicas, estructuras anatómicas, acciones, tareas o áreas de la vida relacionadas entre sí" (Organización Mundial de la Salud [OMS] \& Organización Panamericana de la Salud [OPS], 2001, p. 3). Esta clasificación permite determinar la funcionalidad, la discapacidad y la salud de los lesionados (Organización Mundial de la Salud [OMS] \& Organización Panamericana de la Salud [OPS], 2001, p. 4). Se debe tener en cuenta que los accidentes de tránsito, no solo dejan lesiones físicas, sino que pueden presentarse lesiones de orden sensorial y cognitivas. Por esto, adicional al diagnóstico, es nece- 
sario abordar la situación desde un enfoque que integre los diferentes ámbitos desde el apoyo, el modelo social, la familia y los miembros de su comunidad, y de esta forma, se logre la adaptación a las actividades cotidianas y de participación social

Los discapacitados asociados a la accidentalidad vial deben ser evaluados para definir el concepto, el tipo y el grado de discapacidad, por lo que es necesario contar con una visión integral del fenómeno para conocer las múltiples variables al momento de definirla, tales como las posibles deficiencias estructurales y funcionales hasta factores sociales, personales, económicos y culturales; por tanto, es innegable que deben practicarse diagnósticos, estudios y tratamientos para atender a las personas con esta discapacidad asociada, además porque se debe definir si es permanente o transitoria, porque de esto depende el tipo de enfoque con el que se aborde; es decir, el biopsicosocial, el multidimensional, el dinámico-integral.

$\gg$ El enfoque biopsicosocial en discapacidad considera que el bienestar de las personas depende de la integración de los factores biológicos, psicológicos y sociales en el tratamiento de la discapacidad. Este enfoque tiene sus orígenes en el año de 1977 en la Universidad Rochester sustentado por el psiquiatra George Libman Engel quien propone que se debe incluir científicamente el dominio humano en la experiencia de la enfermedad (Vanegas \& Gil, 2007, p. 58). Este modelo considera el fenómeno como un problema de origen social que debe estar centrado en la completa integración de las personas a la sociedad y se concretiza en las condiciones construidas en el contexto social y en el manejo de la responsabilidad social y colectiva. Según Vanegas \& Gil (2007, p. 59). corresponde a la sociedad generar las modificaciones ambientales necesarias para la participación de las personas en condición de discapacidad en todos los ámbitos de la vida social. Con este enfoque se fomenta una visión coherente de las dimensiones de la salud desde lo biológico, lo individual y lo social.

$\gg$ El enfoque multidimensional se centra específicamente en la discapacidad intelectual basado en un modelo multidimensional del funcionamiento humano, entendido como aquella actividad vital y participativa. Este enfoque está conformado por las siguientes dimensiones: habilidades intelectuales y conducta adaptativa; esto es, habilidades conceptuales, sociales y prácticas; participación, interacción y roles sociales; salud y contexto (American Association on Intelectual and Developmental Disabilities [AAIDD], 2010).

$\gg$ El enfoque dinámico-integral conceptualiza al ser humano y su contexto desde una perspectiva sistémica, dinámica y transdimensional, lo que sustenta aspectos teóricos y prácticos de la educación, la salud y la acción social. Este modelo reconoce que el ser humano es único y simultáneamente físico - molecular, biológico, emocional, cognitivo y espiritual; por tanto, no debe fragmentarse. Estudiar el tema de la discapacidad con este enfoque compromete la colaboración de profesionales de diferentes saberes, como la educación, la medicina, la psicología, la economía, la arquitectura, el derecho, entre otros.

A raíz de la creciente alza en la discapacidad asociada a los accidentes de tránsito que se vienen registrando en por la OMS y la OPS se han formulado y ejecutado planes de acción para los países con el fin de reforzar la vigilancia, la prevención y la rehabilitación para que las personas con discapacidad mejoren su calidad de vida. De igual forma, es notorio que hay avances en la caracterización de personas con discapacidad, y esto se refleja en la diversidad de enfoques conceptuales, metodológicos y en los instrumentos de registro de la información que se han aplicado. Los accidentes de tránsito se han convertido en un problema de salud pública que va en aumento, y por esto, las discapacidades asociadas a este problema determinan la complejidad que reviste, no solo como fenómeno es sí mismo, sino también por las diversas intervenciones para superar las desventajas de acuerdo con el contexto. "En este sentido, si bien se requiere profundizar en el diagnóstico de las causas...se requiere avanzar en el tratamiento de las consecuen- 
cias...discapacitantes que generan los traumatismos causados por el tránsito" (Organización Panamericana de la salud [OPS], 2011, p. 4).

En el estudio de tipo descriptivo titulado "Discapacidad de origen vial en Medellín", Cabrera \& Salazar $(2016$, p.3) exponen que los incidentes viales son la segunda causa de muerte violenta en Colombia y que los "Actores viales, motorizados o no, que sobreviven a incidentes viales graves tienen una amplia probabilidad de quedar con discapacidades...Se estima que, en Colombia, por cada muerte vial, hay 100 lesionados viales, 1 muy grave con secuelas severas, y de 6 a 9 más con discapacidades de grado moderado a leve". Según Cabrera \& Salazar (2016, p. 6), este informe constituye un referente porque determinó con base en el Registro de Localización de Personas con Discapacidad -RLCPCD- al 31 de diciembre de 2016, que en Medellín 3178 personas estaban en condición de discapacidad por accidentes de tránsito, de los cuales el 71\% eran varones, un $25 \%$ tenían 36 años o menos y un $50 \%$ hasta 50 años o menos). Esto refleja las condiciones de movilidad y de trabajo de las personas que puede incrementar el riesgo en las vías, lo que aumenta la probabilidad de sufrir una discapacidad por incidente vial (Cabrera \& Salazar, 2016, p. 23).

A pesar de los esfuerzos de organismos internacionales, nacionales y locales por tener una mirada transversal, con un enfoque social y de derechos humanos en materia de discapacidad asociada a la accidentalidad vial, es innegable que los costos y barreras que supone la condición de discapacidad, inciden en el empobrecimiento, la escasa accesibilidad y en la vulneración de derechos, lo cual evidencia que la mayoría de personas con discapacidad de origen vial en Medellín pertenecen a los estratos 2 y 3 y en su mayoría son jóvenes y esto redunda en la pérdida de capacidad laboral e ingresos económicos. Como ejemplo, Cabrera \& Salazar (2016, p. 21 determinan que en el año 2016 en Medellín hubo aproximadamente 27.250 lesionados con diversos niveles de gravedad, y que podrían ser generadores de discapacidad. Igualmente, los recursos que se destinan en el sistema se consideran como gasto público y poco suficientes

Para realizar estudios estadísticos sobre discapacidad se puede apoyar en técnicas de recolección de información como los censos, las encuestas de hogares, los análisis de registros nacionales de personas con discapacidad y la vigilancia epidemiológica. Cada una de estas estrategias arroja información importante y son una base fundamental para la elaboración de planes de acción y focalización de programas y servicios de apoyo. A nivel internacional, la OMS y la OPS, recomiendan establecer políticas para el registro de la información que permita generar indicadores para conocer en qué medida las estrategias de seguridad vial están funcionando. En ese mismo sentido, promover el cumplimiento y vigilancia de las leyes de seguridad vial con actividades de concienciación sobre los costos humanos, sociales y económicos que pueden generar la inseguridad vial y así, enfatizar en la responsabilidad de los Estados de garantizar la vida e integridad de sus ciudadanos, reconociendo que la seguridad vial es una prioridad en materia de salud pública, por tanto, debe haber una política pública encaminada a garantizar una movilidad segura, equitativa, saludable y sustentable pues los accidentes de tránsito, se deben prevenir.

En este escrito se ha mostrado que la inclusión social de las personas con discapacidad, y en el caso específico de la discapacidad asociada a accidentes de tránsito, depende no solo de acciones independientes y particulares sino también de la acción integral del Estado, de la voluntad política de los gobiernos y de la construcción de políticas públicas para mejorar las condiciones de vida de los ciudadanos con la creación de escenarios para el disfrute pleno de los derechos del grupo poblacional con discapacidad. Bajo esta óptica, se plantea una relación entre discapacidad y justicia, para Molina (2005) "la discapacidad es una categoría moral - política y que es la justicia quien exigirá a las instituciones sociales, actores externos y entes gubernamentales (familia, comunidad, estado y sociedad) el reconocimiento y visibilidad de las personas con discapacidad como sujetos políticos" (p. 259). 
Por esto, a pesar de los estudios y propuestas para el abordaje de la problemática siempre estará la necesidad de agregar nuevos elementos para el análisis crítico y el aporte a su conocimiento. Es así como, aquellas personas con discapacidad asociada a accidentalidad vial son convocadas por la institucionalidad para que desde nuevas reflexiones en torno al tema puedan desentrañar los universos simbólicos de la dimensión humana y explorar desde su interior y desde sus subjetividades las necesidades, las ideas, las percepciones, las actitudes y las conductas que se tienen por la movilidad vial y la discapacidad. En tanto se tengan en cuenta que con estos parámetros se puede sostener que la discapacidad es una categoría social y política que implica prácticas y luchas por reivindicar los derechos humanos, y con esta forma de entenderla se responsabiliza a la sociedad para que se estructure conforme las necesidades y las aspiraciones de las personas con discapacidad.

Como se indicó al principio de este texto, comprender el fenómeno de la inclusión social implica también la comprensión de la exclusión social como resultado de una sociedad fragmentada que se caracteriza por negar o restringir derechos al conjunto de la población. Desde la fragmentación se encuentran varias exclusiones, en el caso de la discapacidad asociada a accidentes de tránsito se pueden determinar las siguientes, según Garay (2002, citado por Molina, 2005, p. 260): la económica como la incapacidad de generar ingresos suficientes para satisfacer las necesidades básicas; la de capital social, cuando se refiere a los pocos mecanismos de participación social; la de capital humano que alude a la falta de acceso y calidad en los servicios de educación y salud y vulneración de los derechos políticos y civiles como son la dimensión política en donde se excluye a la persona de hacer uso del derecho que tiene como ciudadano. También está la perspectiva de justicia que en materia de política pública de inclusión social de las personas discapacitadas por asocio a accidentalidad vial se convierte en el marco argumentativo para fundamentar la dignidad humana y el derecho a reivindicar la posibilidad de ser diferentes, viabilizar esta reivindicación es prueba de que la sociedad está reaccionando y entiende el sentido de la justicia. Si la sociedad es vista como un sistema equitativo de cooperación, las personas con discapacidad pueden desempeñarse como miembros cooperativos. Para Molina (2005, p. 262) un ciudadano entonces es aquel que puede ser un participante libre e igual, que puede decidir y llevar adelante su plan de vida. En otras palabras, tiene el derecho a tener derechos.

Específicamente en Colombia se busca dar cumplimiento a la Convención Internacional sobre los Derechos de las Personas con Discapacidad realizada por la ONU el 13 de diciembre de 2006 para promover y proteger los derechos y la dignidad de las personas e impulsar el desarrollo social y la defensa de los derechos desde la articulación de las recomendaciones de la Comisión de Derechos Humanos y de la Comisión de Desarrollo Social. Esta Convención reconoce a la familia como unidad colectiva natural y fundamental de la sociedad, y quien debe proteger y acompañar a la persona con discapacidad. Solo mediante el cumplimiento de estos compromisos, entre el Estado, la sociedad y la familia se ayudará a disminuir la profunda desventaja social de las personas con discapacidad, se promoverá su participación y la igualdad de oportunidades en los ámbitos económico, civil, político, social y cultural. Conforme Gómez \& González (2008, p. 5) para lograr hacerle frente a la propuesta de la Convención, se realizó un registro donde se observan las necesidades del grupo poblacional con discapacidad en el territorio nacional para orientar la gestión pública, este instrumento muestra los factores asociados a la exclusión social con lo cual se busca generar políticas de intervención en discapacidad en el país. Con este registro se analizan tres dimensiones para dar respuesta desde lo territorial:

$\Rightarrow$ la primera dimensión es la personal o corporal y factores personales; se orienta al ser y se analizan las variables relacionadas con las particularidades de las personas, las deficiencias en sus estructuras o funciones corporales, indaga sobre las causas de la discapacidad y las características 
demográficas. Como respuesta a esto, los entes territoriales han diseñado planes y programas de promoción de espacios protectores y de prevención de la discapacidad que se encuentran en el Plan Nacional de Salud pública y en los planes nacionales y municipales de seguridad vial.

$\gg$ La segunda dimensión es la individual, orientada desde el hacer, evalúa las limitaciones de las personas discapacitadas en su desempeño en la vida cotidiana. La respuesta a esta dimensión es responsabilidad de las EPS, de igual forma se requiere de los bancos de ayudas técnicas y de la estrategia de Rehabilitación Basada en Comunidad -RBC -; en todo caso, el Estado debe hacer seguimiento y control a la cobertura y calidad de los servicios.

$\gg$ La tercera dimensión es la social, desde el estar y el tener; aquí se describen los factores del entorno que afectan la participación de las personas con discapacidad en las mismas condiciones de las demás; por tanto, se analizan las características de la vivienda como el estrato, los servicios públicos, el tipo y la forma. Para Gómez \& González (2008, pp.12-13) la respuesta está encaminada hacia las acciones inclusivas que consisten en identificar las barreras actitudinales y físicas, fortalecer los canales de comunicación, propiciar el disfrute de los derechos como la salud y la educación, promover la participación de las personas discapacitadas y de sus familias mediante las veedurías ciudadanas y en la planificación territorial

Estas dimensiones se encuentran incluidas en la discapacidad, en este caso asociada a los accidentes viales por medio de la Política Pública Nacional de Discapacidad e Inclusión Social - PPDIS - para el periodo 2013-2022, cuyo fin es "asegurar el goce pleno de los derechos y el cumplimiento de los deberes de las personas con discapacidad, sus familias y cuidadores" (Ministerio de Salud y Protección Social, 2018b). Estos derechos se garantizan en la medida en que se comprendan las dinámicas y las lógicas de los territorios; es decir, se parte de las características físicas, económicas, culturales y políticas que componen sus particularidades, por esta razón, la política pública debe adaptarse a estas, "teniendo presente que el vínculo nación - territorio es el que permite que toda la sociedad colombiana, desde su diversidad, transite por la misma vía para el logro de la inclusión plena de las personas con discapacidad" (Ministerio de Salud y Protección Social, 2018a).

Como apoyo a lo anterior, se cuenta con otros documentos que contienen los lineamientos generales para la implementación de una política pública donde se detallan los conceptos y la metodología para su implementación de forma participativa y concertada. Como son el caso de ley 1618 de 2013 y el Plan Nacional de Seguridad Vial y el Plan de Movilidad Segura del Municipio de Medellín.

El tratamiento del tema de la discapacidad y la inclusión social se encuentran en la Ley Estatutaria 1618 de 2013 cuyo objeto es "garantizar y asegurar el ejercicio efectivo de los derechos de las personas con discapacidad, mediante la adopción de medidas de inclusión, acción afirmativa y de ajustes razonables" e introduce una definición de las "personas con y/o en situación de discapacidad" en su Art 2, numeral 1.L 1618/2013 como aquellas que "tengan deficiencias físicas, mentales, intelectuales o sensoriales a mediano y largo plazo que, al interactuar con diversas barreras incluyendo las actitudinales, puedan impedir su participación plena y efectiva en la sociedad, en igualdad de condiciones con las demás". Y en el Art 2, numeral 2. L 1618/2013 presenta el concepto de inclusión social.

un proceso que asegura que todas las personas tengan las mismas oportunidades, y la posibilidad real y efectiva de acceder, participar, relacionarse y disfrutar de un bien, servicio o ambiente, junto con los demás ciudadanos, sin ninguna limitación o restricción por motivo de discapacidad, mediante acciones concretas que ayuden a mejorar la calidad de vida de las personas con discapacidad. 
El Plan Nacional de Seguridad Vial 2011-2021 en su diagnóstico señala los factores que han incidido en Colombia para que los indicadores en materia de seguridad vial estén en rojo:

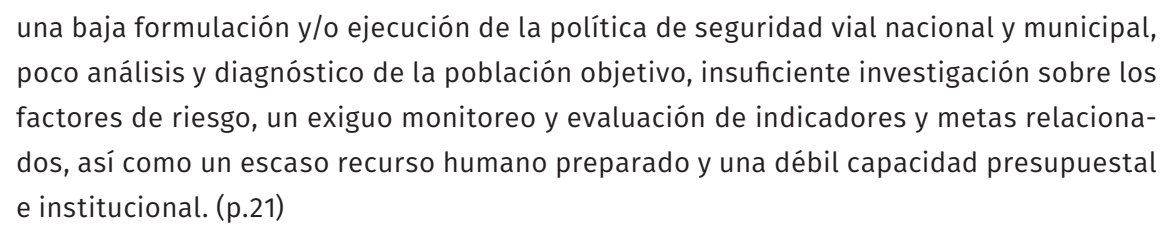

Presenta algunos indicadores de accidentalidad vial: los conocimientos de la norma y la señalización, las actitudes, la pericia, los errores, los lapsus, la infraestructura y las decisiones personales. También, la falta de coordinación entre las autoridades, la no finalización de los procesos iniciados por contravenciones de tránsito, el otorgamiento de licencias de tránsito y las metodologías de enseñanza que son basadas, exclusivamente, en guías. El Plan estratégico seguridad vial Medellín (2014-2020, p.5), se elaboró conforme un diagnóstico que permitió la generación de programas y acciones para avanzar en materia de movilidad segura, las áreas de intervención son: 1. Fortalecimiento de la gestión y coordinación interinstitucional. 2. Comportamientos, hábitos y conductas seguras de los usuarios de las vías. 3. Control y supervisión sobre los vehículos y equipos de seguridad para los usuarios de las vías. 4. Planeación, construcción y mantenimiento de la infraestructura vial segura para los usuarios de las vías. 5. Atención oportuna y profesional de los lesionados durante y después de los hechos de tránsito.

No obstante, en este Plan no se cuenta con suficiente información sobre la discapacidad asociada a los accidentes de tránsito conforme las características sociodemográficas, clínicas y de acceso a los servicios de inclusión de los sujetos involucrados, lo que implica que este tipo de eventos no refleje la eficacia jurídica de la normatividad que está vigente en el país. Además, es necesario tener presente los años de vida saludables que, debido a los accidentes de tránsito, se han perdido por la ocurrencia de lesiones que generan discapacidades asociadas a este hecho en usuarios de la vía en la ciudad de Medellín.

\section{CONCUSIONES}

Una de las estrategias fundamentales de los países para hacerle frente a la exclusión social ha sido la creación de políticas públicas orientadas a la construcción de escenarios democráticos e incluyentes en los ámbitos sociales, económicos, políticos y culturales que hacen su presencia desde las generalidades, las definiciones y los conceptos que constituyen el fundamento de las actuaciones frente a los temas de la inclusión social. La discapacidad asociada a la accidentalidad vial no es ajena pues se debe reconocer que la evolución de su abordaje obedece a los cambios que la sociedad debe plantear como retos y que exigen un viraje en la forma en la que son asumidos por la sociedad en concurrencia con los estamentos del Estado encargados de este fenómeno.

La inclusión social y la condición de discapacidad están ligadas al reconocimiento de los derechos humanos donde la vida digna es el objetivo que prima cuando de luchas y procesos de reivindicación se trata. Entonces, las transformaciones en materia de derechos dan cuenta de los intereses, percepciones, necesidades y posibilidades de quienes, por diferentes razones, se ven afectados en el caso de la discapacidad

asociada a la accidentalidad vial. Es pertinente reconocer que la discapacidad es una condición en que como sujetos vulnerables requiere de procesos para el reconocimiento y cumplimiento de los derechos. En este orden de ideas, con la inclusión social lo que se persigue es que haya redistribución y reconocimiento con participación en condiciones de igualdad real y material y con dignidad. Esto no indica homogeneizar 
a la población, sino que, de acuerdo con los valores y aspiraciones de los sujetos, pueda haber una promoción social encaminada al bienestar de los ciudadanos, pues debe entenderse que la inclusión social parte de admitir que los sujetos con sus dimensiones, son y deben ser diferentes, por tanto, los programas o planes deben ser congruentes con esta premisa.

En el caso de Medellín, las respuestas a la problemática de la discapacidad asociada a la accidentalidad vial permiten planear, implementar y evaluar la normatividad en esta problemática para lograr la inclusión social de la población en situación de discapacidad asociada a la accidentalidad vial por medio del fomento de diferentes acciones de prevención que se orienten a determinar los eventos ocurridos como consecuencia de los accidentes de tránsito y otros eventos de salud que muestran la necesidad de propiciar los ámbitos de integración y exclusión, los enfoques y los modelos de atención.

\section{REFERENCIAS BIBLIOGRAFICA}

American Association on Intellectual and Developmental Disabilities [AAIDD]. (2010). Discapacidad intelectual: definición, clasificación y sistemas de apoyo. [Entrada de blog]. Descargado: agosto 4 de 2018. En: http://cosmonautassinbarreras.blogspot.es/1409545555/aaidd-enfoque-multidimensional-de-comprension-de-la-discapacidad-intelectual/

Bobbio, N. (2007). Teoría General del Derecho. Bogotá: TEMIS.

Cabrera, G., Velásquez N. \& Valladares M. (2009). Seguridad vial, un desafío de salud pública en la Colombia del siglo XXI. En: Revista Facultad Nacional de Salud Pública, 27 (2). 218-225.

Cabrera, G. \& Salazar, E. (2016). Discapacidad de origen vial en Medellín. Descargado: agosto 4 de 2018. En:

https://static1.squarespace.com/static/566f00bcdf40f36736797abd/t/59c8fc1759cc68469d1 1e695/1516122577841/Informe+de+Discapacidad+Vial+MEDELLI\%CC\%81N.pdf

Camacho, J. (2014). Exclusión social. Eunomía. En: Revista en cultura de la legalidad, (7), 208-214.

Espinoza, O. \& Francisco J. (2008). La "nueva pobreza": elementos para su comprensión. El caso de Valparaíso. V Jornadas de Sociología de la UNLP. Universidad Nacional de La Plata. Facultad de Humanidades y Ciencias de la Educación. Departamento de Sociología, La Plata.

Garay L. (2002). Repensando a Colombia. Hacia un nuevo contrato social, PNUD - ACCI. Bogotá.

Garlan, R. (1995). the eye of the beholder deformity \& desabilityin the graecoroman world, duckworth, London.

Gómez, J. \& González, C. (2008). Discapacidad en Colombia: reto para la inclusión en capital humano. Situación nacional. Tomo 1. Bogotá: Fundación Saldarriaga Concha.

Hernández Aja, A. (2011). Análisis urbanístico de barrios vulnerables en España. En: Observatorio de la Vulnerabilidad Urbana, Instituto Juan de Herrera y Ministerio de Fomento, Madrid.

Holzmann, R. \& Jorgensen, S. (2003). Manejo social del riesgo: un nuevo marco conceptual para la protección social y más allá. En: Revista de la Facultad Nacional de Salud Pública. 21 (1), 73-106. Descargado: agosto 11 de 2018. En: http://www.redalyc.org/articulo.oa?id=12021106

Lahera, E. (2002). Introducción a las políticas públicas. Chile: Fondo de cultura económica.

L.1618/2013, Art 5

Ministerio de Salud y Protección Social de Colombia (2018a). Discapacidad. Descargado: septiembre 1 de 2018. En: https://www.minsalud.gov.co/proteccionsocial/Paginas/DisCAPACIDAD.aspx

Ministerio de Salud y Protección Social de Colombia (2018b). Política pública nacional de discapacidad e 
inclusión social. Descargado: septiembre 1 de 2018. En: https://www.minsalud.gov.co/proteccionsocial/promocionsocial/Discapacidad/Paginas/politica-publica.aspx

Ministerio de transporte. (2011). Plan Nacional de Seguridad Vial (2011-2016). Descargado: 25 de agosto de 2017. En: https://www.mintransporte.gov.co/descargar.php?id=1205

Ministerio de transporte. (2015). Plan Nacional de Seguridad Vial (2011-2021). Descargado: 25 de agosto de 2017. En: https://www.mintransporte.gov.co/Publicaciones/plan_nacional_de_seguridad_vial

Molina Béjar, R. (2005). La discapacidad y su inclusión social: un asunto de justicia. Revista de la Facultad de Medicina, 53(4), 259-262. Descargado: November 03, 2018. From: http://www.scielo.org.co/scielo. php?script=sci_arttext\&pid=S0120-00112005000400007\&lng=en\&tlng=es

Municipio de Medellín. (2014). Informe anual de accidentalidad. Descargado: 25 de agosto de 2017. En: https://www.medellin.gov.co/movilidad/jdownloads/Cifras\%20y\%20Estudios/Accidentalidad/Cifras\%20de\%20accidentalidad\%20Anual/informe_anual_2014.pdf

Naciones Unidas [UN] (2006). Convención sobre los derechos de las personas con discapacidad. Descargado: agosto 25 de 2018. En: https://www.un.org/esa/socdev/enable/documents/tccconvs.pdf

Organización Mundial de la Salud [OMS] \& Organización Panamericana de la Salud [OPS] (2001). Clasificación Internacional de funcionamiento, de la discapacidad y de la salud. Descargado: agosto 25 de 2018. En: http://www.deis.cl/clasificacion-internacional-del-funcionamiento-de-la-discapacidad-y-de-la-salud-cif/

Organización Mundial de la Salud [OMS] \& Banco Mundial (2011). Informe mundial sobre la discapacidad. Descargado: agosto 25 de 2018. En: https://www.who.int/disabilities/world_report/2011/es/

Organización Panamericana de la Salud [OPS] \& Organización Mundial de la Salud [OMS] (2004). Día Mundial de la Salud 2004: Seguridad vial. Descargado: agosto 25 de 2018. En: http://www1.paho.org/spanish/dd/pin/whd04_features.htm

Organización Panamericana de la Salud [OPS] (2011). Traumatismos causados por el tránsito y discapacidad. Descargado: agosto 25 de $2018 . \quad$ En: https://www.paho.org/hq/dmdocuments/2012/accidentes-discapacidad.pdf

Sandoval, B. (2016). ¿Inclusión en qué? Conceptualizando la inclusión social. En: Ehquidad International Welfare Policies and Social Work Journal, 5, 71-108. doi.10.15257/ehquidad.2016.0003.

Sen, A. (2000). El desarrollo como libertad. En: Gaceta Ecológica, (55), 14-20.

Subirats I Humet, J. (Dir.), Gomà Carmona, R. \& Bruguè Torruella, J. (Coords.). Fundación BBVA. (2005). Análisis de los factores de la exclusión social. En: Catalunya: Institut de Govern I Poliques Publiques. Descargado: septiembre 15 de 2018. En: https://www.fbbva.es/publicaciones/analisis-de-los-factores-de-exclusion-social/

Toboso, M. \& Arnau, M. (2008). La discapacidad dentro del enfoque de capacidades y funcionamientos de Amartya Sen. Araucaria. En: Revista Iberoamericana de Filosofía, Política y Humanidades, 10 (20), 64-94.

Vanegas, J. \& Gil, L. (2007). La discapacidad, una mirada desde la teoría de sistemas y el modelo biopsicosocial. Hacia la promoción de la salud, 12, 51-61. Descargado: septiembre 15 de $2018 . \quad$ En: http://www. scielo.org.co/pdf/hpsal/v12n1/v12n1a04.pdf

Young, I. (2000). Inclusion and Democracy. Oxford: Oxford University Press. 